\title{
Universality of liquid-gas Mott transitions at finite temperatures
}

\author{
Stefanos Papanikolaou, ${ }^{1}$ Rafael Monteiro Fernandes, ${ }^{2,3}$ Eduardo \\ Fradkin, ${ }^{1}$ Philip W. Phillips, ${ }^{1}$ Joerg Schmalian, ${ }^{2}$ and Rastko Sknepnek ${ }^{2}$ \\ ${ }^{l}$ Department of Physics, University of Illinois at Urbana-Champaign, 1110 W. Green St., Urbana, IL 61801-3080 \\ ${ }^{2}$ Ames Laboratory and Department of Physics and Astronomy, Iowa State University Ames, IA 50011, USA \\ ${ }^{3}$ Instituto de Física "Gleb Wataghin", Universidade Estadual de Campinas, \\ and Laboratório Nacional de Luz Síncrotron,Campinas, SP, Brazil
}

(Dated: November 10, 2018)

\begin{abstract}
We explain in a consistent manner the set of seemingly conflicting experiments on the finite temperature Mott critical point, and demonstrate that the Mott transition is in the Ising universality class. We show that, even though the thermodynamic behavior of the system near such critical point is described by an Ising order parameter, the global conductivity can depend on other singular observables and, in particular, on the energy density. Finally, we show that in the presence of weak disorder the dimensionality of the system has crucial effects on the size of the critical region that is probed experimentally.
\end{abstract}

Although band theory predicts that a system of electrons in a solid with one electron per site (unit cell) should be metallic, such a system ultimately insulates [1, 2] once the local electron repulsive interactions exceeds a critical value. The onset of the insulating state, the Mott transition, arises from the relative energy cost of the on-site Coulomb repulsion $U$ between two electrons on the same lattice site, and the kinetic energy, represented by the band width $W$. Then, the transition is governed solely by the ratio of $U / W$. At $T=0$, it is often the case that symmetries of the microscopic system, associated with charge, orbital or spin order, may be broken in the Mott insulating state. However, at sufficiently high temperatures $T$, or in strongly frustrated systems, no symmetry is broken at the finite- $T$ Mott transition. Then, the transition is characterized by paramagnetic insulating and metallic phases, whose coexistence terminates at a second-order critical point, depicted in Fig. 1(a) In this paper, we are concerned with the universal properties of this classical critical point [3], as revealed by a series of apparently conflicting experiments on $\left(\mathrm{Cr}_{1-x} \mathrm{~V}_{x}\right)_{2} \mathrm{O}_{3}$ [4] and organic salts of the $\kappa-$ ET family [5].

Since no symmetry is broken at the finite- $T$ Mott transition, in a strict sense there is no order parameter. Nonetheless, experimental [4, 5], as well as theoretical evidence [6, 7] suggest that the transition is in the Ising universality class, similar to the liquid-vapor transition. For example, Castellani et al. [6] constructed an effective Hamiltonian for this problem, and proposed that double occupancy should play the role of an order parameter for the Mott transition. On the insulating side, doubly occupied sites are effectively localized, but in the metal, they proliferate. A Landau-Ginzburg analysis [7] provided further evidence for a non-analyticity in the double occupancy at a critical value of $U / W$ that defines a Mott transition. Ising universality follows immediately because double occupancy, $\left\langle n_{i \uparrow} n_{i \downarrow}\right\rangle$, is a scalar local density field.

Experimentally, the universality of the Mott critical point is typically probed by some external parameter, such as pressure, which can tune the ratio $W / U$. Measurements of the conductivity, $\Sigma$, on $\left(\mathrm{Cr}_{1-x} \mathrm{~V}_{x}\right)_{2} \mathrm{O}_{3}$ [4] found that away from the critical point, the exponents defined through

$$
\begin{aligned}
\Delta \Sigma(t, h=0) & =\Sigma(t, h=0)-\Sigma_{c} \propto|t|^{\beta_{\sigma}}, \\
\Delta \Sigma(t=0, h) & \propto|h|^{1 / \delta_{\sigma}}, \\
\partial \Sigma(t, h) /\left.\partial h\right|_{h=0} & \propto|t|^{-\gamma_{\sigma}},
\end{aligned}
$$

have mean-field Ising values, $\beta_{\sigma} \simeq 1 / 2, \gamma_{\sigma} \simeq 1$ and $\delta_{\sigma} \simeq 3$. Here, $t=\left(T-T_{c}\right) / T_{c}$ and $h=\left(P-P_{c}\right) / P_{c}$, with $\left(\Sigma_{c}, T_{c}, P_{c}\right)$ denoting the corresponding values at the critical endpoint. Close to the critical region, Limelette et al. [4] observed a drift to the critical exponents of the 3D Ising universality class. Mean field behavior is also seen in $\mathrm{NiS}_{2}$ [8].

However, similar pressure measurements [5] on the quasi2D organic salts of the $\kappa$-ET family appear to challenge the view that the Mott transition is in the Ising universality class. In this material, Kagawa et al. [5] found that their data is described by the exponents $\beta_{\sigma} \simeq 1, \gamma_{\sigma} \simeq 1$, and $\delta_{\sigma} \simeq 2$, which do not seem to be consistent with the known exponents of the 2D Ising model whose exponents are [9] $\beta=\frac{1}{8}$, $\gamma=\frac{7}{4}$ and $\delta=15$. Since the exponents obey the scaling law $\gamma_{\sigma}=\beta_{\sigma}\left(\delta_{\sigma}-1\right)$, it was proposed that the Mott transition is in a new, as yet unknown universality class. The situation is further complicated by thermal expansion measurements [10] that claim to measure the heat capacity exponent $\alpha$ and find $0.8<\alpha<0.95$. This result is not only in sharp contrast to the expectation for an Ising transition (where $\alpha=0$ for $d=2$ ), it also strongly violates the scaling law $\alpha+2 \beta_{\sigma}+\gamma_{\sigma}=2$, if one uses the exponents of Ref. [5].

In this paper, we present a unified phenomenological description of all of these experimental facts within an Isingtype model, and resolve the issue of the universality class of the Mott transition. A complete description of these experiments requires to take into account that the conductivity depends on all possible singular observables of the associated critical system, and not just on the thermodynamic order parameter associated with the phase transition. Similar considerations were made in magnetic systems near the Curie temperature [11, 12, 13], to explain the critical exponent of the conductivity along the coexistence curve. In that case, a symmetry of the microscopic definition of the conductance 


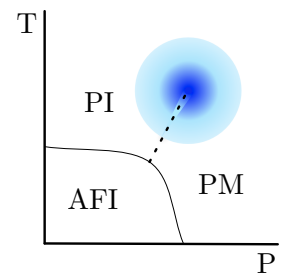

(a)

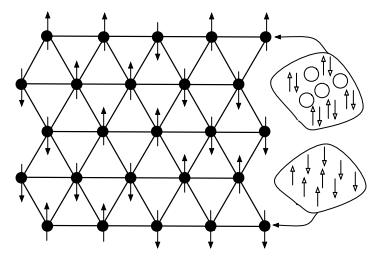

(b)
FIG. 1: (a) Typical phase diagram of Mott transitions as a function of pressure $P$ and temperature $T$. At low $T$ and $P$, a Néel antiferromagnetic insulator (AFI) appears; it becomes a paramagnetic insulator (PI) if $T$ increases, or a paramagnetic metal (PM) if $P$ increases. Dashed line: first-order transition ending at a liquid-gas critical point. Full lines: continuous phase transition to the ordered state. Colored regions: critical (dark) and mean-field (light) regimes of the critical point. (b) An Ising configuration on the triangular lattice. Up (down) spins correspond to conducting (insulating) grains of linear size of the order of the system's dephasing length.

prevented any coupling of the global conductivity to odd moments of the order parameter, along the coexistence line. Even though similar in spirit, the situation here is much different. Starting from an effective microscopic model near an Ising critical point, we show that: 1) the conductivity typically depends on all possible singular thermodynamic observables of the system, namely the order parameter and energy density of the Ising model; 2) when the coupling to the energy density dominates, there exists a large regime around the critical point, where the critical exponents for the conductivity are $\left(\beta_{\sigma}, \gamma_{\sigma}, \delta_{\sigma}\right)=\left(1, \frac{7}{8}, \frac{15}{8}\right)$, that agree (within the error bars) with the findings of Kagawa et al. [5], and the corresponding mean-field exponents are $\left(\beta_{\sigma}^{M F}, \gamma_{\sigma}^{M F}, \delta_{\sigma}^{M F}\right)=\left(1, \frac{1}{2}, \frac{3}{2}\right)$; 3 ) a crossover to Ising exponents is obtained in the order parameter dominated regime as seen in Refs. [4, 8]; 4) in the presence of disorder the Mott critical point ultimately belongs to the random-field Ising model universality class, and therefore the dimensionality of the system under study is even more important for specifying its critical properties.

In order to resolve the discrepancies raised by these experiments, we consider the behavior of the conductivity of the system near the Mott critical point, assuming that it belongs to the 2D Ising universality class. Rather than starting from a microscopic picture, e.g. a Hubbard model, we consider a coarse-grained model with the correct symmetries in which the physics of the relevant transport degrees of freedom is captured. In this picture, one defines coarse-grained regions, of linear size of the order of the dephasing length $l_{\phi}$ of the system, which are either insulating or conducting. Along these lines, we consider an Ising model on a 2D lattice (cf. Fig. 1(b). Near the critical point, where the correlation length for density fluctuations $\xi$ diverges, it is expected that the relevant degrees of freedom behave classically. The Ising variables $S_{i}$ on each lattice site represent the fluctuating density of mobile carriers on microscopic "grains" of linear size of the order of the dephasing length $l_{\phi}$, which are conducting
$\left(S_{i}=+1\right)$, or insulating $\left(S_{i}=-1\right)$. The Hamiltonian is

$$
\beta H=-\frac{1}{T} \sum_{\langle i j\rangle} S_{i} S_{j}+\frac{h}{T} \sum_{i} S_{i},
$$

where $T$ is the temperature, $P$ and $P_{c}$ are the pressure and the critical pressure, respectively, and $h \propto P-P_{c}$ plays the role of the Ising magnetic field. This model is expected to describe the physics near the critical point, where $\xi \gg l_{\phi}$. In this limit, all other interactions beyond nearest-neighbor are irrelevant. Near the critical point, the most singular effect of the pressure is described by a coupling to the order parameter.

To relate the order parameter fluctuations to the transport properties we will define an associated resistor network for this model, an approach that has been successfully used in other strongly correlated systems [14, 15]. Let $\sigma_{C}$ and $\sigma_{I}$ be the local conductivities of the conducting and insulating regions, respectively. We define the bond conductance of the network model simply by adding these two conductivities in series. The bond conductance has three possible values, depending on the state of each grain, which can both be conducting, both insulating, one conducting and the other insulating. Thus, the conductance of the bond $(i, j)$ has the form

$$
\sigma_{i j}=\sigma_{0}\left(1+g_{m}\left(S_{i}+S_{j}\right)+g_{\epsilon} S_{i} S_{j}\right) .
$$

Even in this toy model, the microscopic conductivity, $\sigma_{i j}$, couples both to the order parameter, $S_{i}$, and to the energy density, $S_{i} S_{j}$, of the Ising model with naturally large couplings, $g_{m}$ and $g_{\epsilon}$, defined in Eq. (3). More specifically, we find that $\sigma_{0}=\frac{1}{4}\left(\sigma_{C}+\sigma_{I}\right)+\frac{\sigma_{C} \sigma_{I}}{\sigma_{C}+\sigma_{I}}, g_{m}=\frac{\sigma_{C}-\sigma_{I}}{4 \sigma_{0}}$, and $g_{\epsilon}=\frac{\left(\sigma_{C}-\sigma_{I}\right)^{2}}{4 \sigma_{0}\left(\sigma_{C}+\sigma_{I}\right)}$. At high contrast, $\sigma_{C} \gg \sigma_{I}$, we get $g_{m} \simeq g_{\epsilon} \simeq 1$, whereas, at low contrast, $\left|\sigma_{I}-\sigma_{C}\right| \ll \sigma_{C}$, we get $g_{\epsilon}<g_{m} \rightarrow 0$.

The conductivity of the 2D Ising model we described is a non-trivial quantity to compute. As it was shown in the simpler case of the random resistor network (RRN) [16], networks of bonds with conductance $\sigma_{C}\left(\sigma_{I}\right)$ chosen randomly with probability $p$ and $1-p$, the global conductivity becomes non-zero as soon as an infinite percolating and conducting cluster emerges in the system. When $\sigma_{I}=0$, the critical exponent $\beta_{\sigma}$ of the conductivity is non-trivially related to the fractal properties of the incipient infinite conducting cluster. This exponent is larger than unity for random uncorrelated networks and larger than the exponent of the order parameter, because dangling bonds of the infinite cluster do not contribute to the conductivity. On the other hand, it becomes much less than unity for correlated networks, and typically very close to the exponent of the order parameter, since the infinite cluster is efficiently connected with few dangling bonds.

On the other hand, when $\sigma_{I}>0$, a conducting cluster is less distinguishable from that of an insulating one, and the complex effects coming from the fractal cluster boundaries are smeared out. In the context of RRN, the percolation transition is not seen in the behavior of the conductivity, which seems to show just a crossover. If the contrast is low, $\sigma_{I} \simeq \sigma_{C}$, 


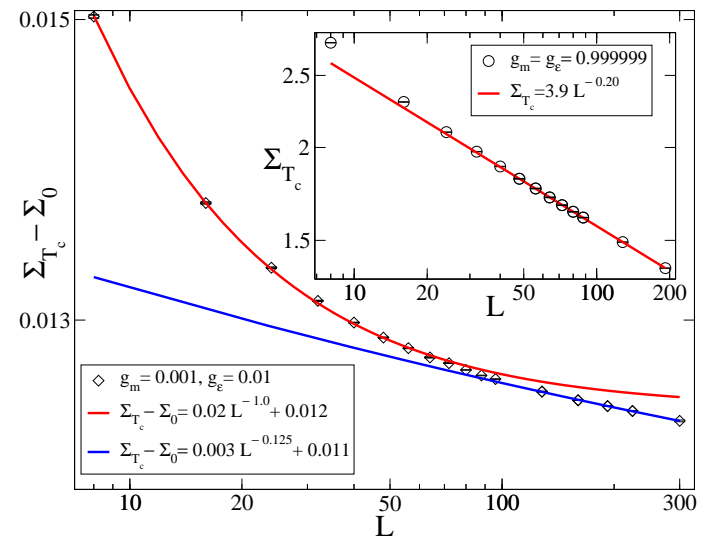

FIG. 2: Crossover behavior of the conductivity at finite contrast: the energy density (order parameter) dominates at short (long) length scales. Inset: Fractal scaling at large contrast.

the actual conductivity of a single bond between sites $i, j, \Sigma$, should depend only on local observables, and we can formally expand it in powers of $g_{m}$ and $g_{\epsilon}$ [17],

$$
\Sigma=\sigma_{0}+g_{m}\left\langle\left(S_{i}+S_{j}\right)\right\rangle+g_{\epsilon}\left\langle S_{i} S_{j}\right\rangle+\ldots,
$$

where the ellipsis represents more complex products of local spin operators (weighed by rapidly decaying functions) [17]. Near the Ising critical point, the most singular contribution of the expectation values of multi-spin operators in Eq. 4 is given by the expectation value of the most singular, "primary", operators of the Ising critical point, the order parameter $m$ and the energy density $\epsilon$. Thus, the most singular term of multispin operators with odd (even) number of spins is proportional to the order parameter (energy density). Therefore, within the range of convergence of this expansion,

$$
\Sigma=\Sigma_{0}\left(g_{m}, g_{\epsilon}\right)+f_{m}\left(g_{m}, g_{\epsilon}\right)\langle m\rangle+f_{\epsilon}\left(g_{m}, g_{\epsilon}\right)\langle\epsilon\rangle,
$$

where $\Sigma_{0}, f_{m}, f_{\epsilon}$ are non-universal regular polynomials in $g_{m}$ and $g_{\epsilon}$. Provided that the critical behavior is still controlled by the fixed point theory of the Ising model, the total conductivity should have the structure of Eq. (5). Thus, at finite contrast, Eq. (5) predicts that the actual conductivity is the sum of even and odd components, under the Ising symmetry transformation, $\Sigma=\Sigma_{0}+\Sigma^{\text {even }}+\Sigma^{\text {odd }}$, and it should exhibit a crossover from an energy density dominated behavior at short distances to an order parameter dominated behavior at long distances. The crossover scale is controlled by the relative size of the functions $f_{m}$ and $f_{\epsilon}$ (cf. Fig. 2). This behavior breaks down at high contrast where there is multi-fractal behavior (cf. Inset in Fig, 2] and Ref. [18]).

We can understand the experiments of Refs. [4, 5, 8], if we assume that Eq. (5) applies. The results of Refs. [4, 8] follow by assuming that $f_{m}\left(g_{m}, g_{\epsilon}\right)>f_{\epsilon}\left(g_{m}, g_{\epsilon}\right)$, and the conductivity scales as the order parameter. Conversely, the results of Ref. [5] follow if $f_{\epsilon}\left(g_{m}, g_{\epsilon}\right) \gg f_{m}\left(g_{m}, g_{\epsilon}\right)$, and the conductivity, for an extended regime near the critical point, scales as the energy density of the Ising model. In this case

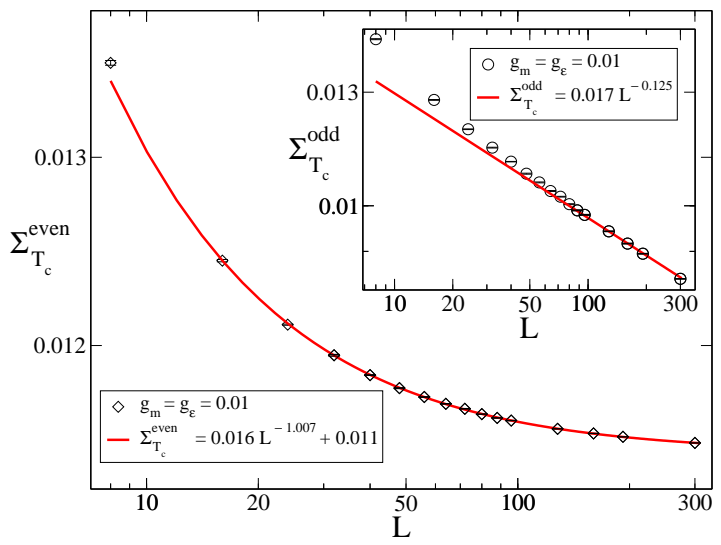

FIG. 3: Monte Carlo data which verify the expected behavior of the conductivity when $g_{m}, g_{\epsilon} \ll 1$. $\Sigma^{\text {even }}$ scales as the energy density (see text). Inset: $\Sigma^{\text {odd }}$ scales as the order parameter.

holds $\Delta \Sigma \propto|m|^{\theta}$, where $\theta=(1-\alpha) / \beta$. Then, it follows that $\beta_{\sigma}=\theta \beta, \delta_{\sigma}=\delta / \theta$ and $\gamma_{\sigma}=\gamma+\beta(1-\theta)$. The resulting critical exponents are $\left(\beta_{\sigma}, \gamma_{\sigma}, \delta_{\sigma}\right)=\left(1, \frac{7}{8}, \frac{15}{8}\right)$, very close to the experimental values. These exponents obey

$$
\gamma_{\sigma}=\beta_{\sigma}\left(\delta_{\sigma}-1\right)
$$

if $\gamma=\beta(\delta-1)$, i.e., the conductivity exponents obey a scaling relation identical to the Ising exponents, in agreement with the experimental verification of this scaling relation in Ref. [5]. In addition, the scaling function obtained by Kagawa et al. [5] only depends on $\beta \delta=\beta_{\sigma} \delta_{\sigma}$, as in our theory.

In order to verify the theoretical picture presented above, we performed Monte Carlo simulations of the 2D Ising model on square and triangular lattices, using the Wolff cluster algorithm [19]. For the calculation of the conductivity, for each Ising configuration we used the Franck - Lobb algorithm [20], or explicitly solved Kirchhoff equations. As expected, we found that at the Ising critical point, for $g_{m}, g_{\epsilon} \ll 1$, the even component of the conductivity $\Sigma^{\text {even }}$ scales as the energy density, while the odd component $\Sigma^{\text {odd }}$ scales as the order parameter (cf. Fig. 3). As $g_{m}, g_{\epsilon}$ approach unity, a slow crossover exists to a fractal regime of the Ising clusters, which is crucial for specifying the critical exponent of the conductivity, consistent with the results of Ref. [18] (cf. Inset in Fig,2])

Refs. [4, 8] report 3D mean-field Ising behavior and a small critical region in $\left(\mathrm{Cr}_{1-x} \mathrm{~V}_{x}\right)_{2} \mathrm{O}_{3}$ and $\mathrm{NiS}_{2}$ under pressure respectively, in contrast to the extended critical region with 2D Ising exponents of Ref. [5]. We can understand these experiments by considering the effects of quenched disorder on an Ising critical point. The difference between a quasi-2D and a $3 \mathrm{D}$ material is a strongly anisotropic Ising interaction along the direction perpendicular to the planes. Disorder that locally favors the localized over the delocalized state or vice versa, corresponds to a random magnetic field, and couples to the order parameter of the Ising transition. This induces density fluctuations. The relevant model for this discussion is the 
anisotropic 3D random-field Ising model (RFIM),

$$
H=-J_{x y} \sum_{\{i j\}_{x y}} S_{i} S_{j}-J_{z} \sum_{\{k l\}_{z}} S_{k} S_{l}+\sum_{i} h_{i} S_{i},
$$

where $h_{i}$ is a random field with variance $\Delta$. For $d=3$, there is a continuous phase transition in the $3 D$ random-field Ising model (3DRFIM) universality class [21] for any anisotropy $J_{x y} / J_{z}$, an irrelevant operator at the 3D RFIM fixed point. However, for large anisotropy and weak disorder, relevant to the quasi-2D organics, there is a large dimensional crossover regime from 2D RFIM behavior, with an exponentially long correlation length, to the narrower 3D RFIM criticality [22]. What changes between the $3 \mathrm{D}$ isotropic materials and the quasi-2D organics is not the universality class, but where the planar correlations become critical. For weak disorder $\Delta \ll J_{x y}$ and strong anisotropy $J_{z} / J_{x y} \ll 1$, the planes are essentially decoupled and 2D-RFIM behavior holds with $\xi_{x y} \gg 1$ in a large region away from the transition point. When $J_{z} \simeq J_{x y}$, the critical region is narrow, and controlled by the 3D RFIM fixed point.

With regards to the thermal expansion measurements that claim to measure the heat capacity exponent $\alpha$, we argue that the authors of Ref. [10] misinterpret their results. The volume change is proportional to the Ising order parameter of the Mott transition, i.e. $\Delta l \propto m$, yielding $l^{-1} d l / d T \propto t^{\beta-1}$. The thermal expansion diverges with exponent $1-\beta=0.875$, consistent with Ref. [10] who find it in the range $0.8-0.95$.

Some major predictions can be drawn from our picture. Firstly, all thermodynamic observables near the Mott critical point should have Ising critical exponents. Secondly, regarding the critical behavior in quasi-2D organic salts [5], in the clean system, the conductivity along the coexistence line should have the same critical exponent $\left(\beta_{\sigma}=1\right)$ in both meanfield and true-critical regimes. This means that the conductivity jump $\Delta \Sigma_{J} \equiv \Sigma\left(T, h=0^{+}\right)-\Sigma\left(T, h=0^{-}\right)$along the coexistence line, which should be proportional to the order parameter, should have distinct mean-field and critical regimes, where $\beta_{\Delta \Sigma_{J}}=1 / 2$ and $\beta_{\Delta \Sigma_{J}}=1 / 8$ respectively. Also, the first-order Mott transition is expected to be broadened by disorder [23, 24]. Thus, instead of a sharp jump in the conductance one should see a continuous change which would become more abrupt for clean systems. The net effect is to make the system spatially inhomogeneous, as in charge ordered phases, stripes and electron nematics [25] and in the manganites [26], which tend to round their phase transitions and replace the first-order transition by an inhomogeneous phase. Thus, hysteretic glassy-like aging effects [27], a problem that has been studied only recently in strongly correlated systems [14, 28], are also expected at this Mott transition.

In conclusion, we have explained under a consistent phenomenological framework the series of experiments that were performed during the last few years on Mott criticality. We showed that the conductivity of a system near a critical point depends on all possible local singular observables of the system, which in the case of interest, are the order parameter and the energy density of the effective Ising model. This description holds when the contrast between conducting and insulating regions is small. Should this not hold, fractal behavior of the incipient infinite clusters at the critical point is crucial for specifying the critical exponents of the conductivity. Finally, disorder affects the effective dimensionality of the system. In particular, we showed that for quasi-2D materials, such as the organic salts in the $\kappa$-ET family, critical fluctuations are expected to be much larger than in a 3D material in an extended regime in the $(P, T)$ plane around the Mott critical point.

Acknowledgements We thank K. Kanoda for discussions. This work was supported in part by the National Science Foundation grants DMR 0442537 (EF) and DMR 0605769 (PP) at UIUC, by the U.S. Department of Energy, Division of Materials Sciences under Award DE-FG02-07ER46453 (EF), through the Frederick Seitz Materials Research Laboratory at UIUC, and the Ames Laboratory, operated for the U.S. Department of Energy by Iowa State University under Contract No. DE-AC02-07CH11358 (JS), and by CAPES and CNPq (Brazil) (RF).

[1] N. F. Mott, Proc. Phys. Soc. A 62, 416 (1949).

[2] W. F. Brinkman and T. M. Rice, Phys. Rev. B 2, 4302 (1970).

[3] This should not be confused with the putative quantum critical scenario of M. Imada, Phys. Rev. B, 72, 075113 (2005).

[4] P. Limelette et al., Science 302, 89 (2003).

[5] F. Kagawa, K. Miyagawa, and K. Kanoda, Nature 436, 534 (2005).

[6] C. Castellani et al., Phys. Rev. Lett. 43, 1957 (1979).

[7] G. Kotliar, E. Lange, and M. J. Rozenberg, Phys. Rev. Lett. 84, 5180 (2000).

[8] N. Takeshita et al., arXiv:0704.0591 (2007), H. Takagi, private communication.

[9] B. M. McCoy and T. T. Wu, The Two-Dimensional Ising Model (Harvard University Press, Cambridge, 1973).

[10] M. de Souza et al., arXiv:cond-mat/0610576v1 (2006).

[11] P. C. Hohenberg and B. I. Halperin, Rev. Mod. Phys. 49, 435 (1977).

[12] M. E. Fisher and J. S. Langer, Phys. Rev. Lett. 20, 665 (1968).

[13] I. Mannari, Phys. Lett. A 26, 134 (1968).

[14] E. W. Carlson et al., Phys. Rev. Lett. 96, 097003 (2006).

[15] J. Burgy et al., Phys. Rev. Lett. 87, 277202 (2001).

[16] D. Stauffer and A. Aharony, Introduction to Percolation Theory (CRC Press, 1991).

[17] J. A. Blackman, J. Phys. C: Solid State Phys. 9, 2049 (1976).

[18] P. J. M. Bastiaansen and H. J. F. Knops, J. Phys. A: Math. Gen. 30, 1791 (1997).

[19] U. Wolff, Phys. Rev. Lett. 62, 361 (1989).

[20] D. J. Franck and C. J. Lobb, Phys. Rev. B 37, 302 (1988).

[21] T. Nattermann, in Spin Glasses and Random Fields, edited by A. P. Young (World Scientific, Singapore, 1998).

[22] O. Zachar and I. Zaliznyak, Phys. Rev. Lett. 91, 036401 (2003).

[23] Y. Imry and M. Wortis, Phys. Rev. Lett. 19, 3580 (1979).

[24] M. Aizenman and J. Wehr, Phys. Rev. Lett. 62, 2503 (1989).

[25] S. A. Kivelson, E. Fradkin, and V. J. Emery, Nature 393, 550 (1998).

[26] E. Dagotto, T. Hotta, and A. Moreo, Phys. Rep. 344, 1 (2001).

[27] J. P. Sethna, K. A. Dahmen, and O. Perković, in Science of Hys- 
teresis, edited by I. D. Mayergoyz and G. Bertotti (Academic Press, London, 2004).
[28] J. Schmalian and P. Wolynes, Phys. Rev. Lett. 85, 936 (2000). 